\title{
Clinical characteristics of cytomegalovirus colitis: a I5-year experience from a tertiary reference
} center

This article was published in the following Dove Press journal:

Therapeutics and Clinical Risk Management

\author{
Puo-Hsien Le ${ }^{1,2}$ \\ Wey-Ran Lin ${ }^{1,3}$ \\ Chia-Jung Kuo ${ }^{1-3}$ \\ Ren-Chin $\mathrm{Wu}^{3,4}$ \\ Jun-Te Hsu ${ }^{3,5}$ \\ Ming-Yao Su ${ }^{1-3}$ \\ Chun-Jung Lin' \\ Cheng-Tang Chiu ${ }^{1-3}$ \\ 'Department of Gastroenterology \\ and Hepatology, Linkou Chang \\ Gung Memorial Hospital, ${ }^{2}$ Taiwan \\ Association for the Study of Small \\ Intestinal Diseases, ${ }^{3}$ College of \\ Medicine, Chang Gung University, \\ ${ }^{4}$ Department of Pathology, \\ ${ }^{5}$ Department of General Surgery, \\ Linkou Chang Gung Memorial \\ Hospital, Taoyuan, Taiwan
}

Background: Cytomegalovirus (CMV) colitis is considered rare in immunocompetent patients. Objective: The predictors of mortality and the differences between immunocompetent and immunocompromised patients with this disease remain unknown. Thus, the aim of this retrospective cohort study was to clarify these issues.

Patients and methods: We enrolled all patients who were histologically diagnosed with CMV colitis between April 2002 and December 2016 in the Linkou Chang Gung Memorial Hospital. Patients were divided into two groups: immunocompetent and immunocompromised, and the differences between them were analyzed to develop in-hospital mortality predictors.

Results: A total of 69 patients (42, immunocompetent; 27 , immunocompromised) were enrolled. The most common symptoms were melena in the immunocompetent group and diarrhea in the immunocompromised group. The in-hospital mortality rate showed no statistically significant difference between the two groups $(26.2 \%$ vs $25.9 \%, P=0.981)$. Early diagnosis was the only significant independent predictor of in-hospital mortality (odds ratio [OR] 1.075, 95\% CI 1.005-1.149, $P=0.035$ ). The cutoff of diagnostic timing was 9 days from admission, derived from the receiver operating characteristic curve using the Youden index.

Conclusion: CMV colitis in immunocompetent patients is markedly more common and fatal than has generally been acknowledged. Being alert to different ways in which this disease can present itself will enable early diagnosis and significantly reduce mortality.

Keywords: cytomegalovirus colitis, immunocompetent, immunocompromised

\section{Introduction}

Cytomegalovirus (CMV) colitis is mostly found in immunocompromised patients (solid organ or hematopoietic stem cell transplant recipients, patients with human immunodeficiency virus [HIV] infection, and immunosuppressive drug users, including steroid or chemotherapeutic agents), and it often has a poorer outcome in adults than in children. ${ }^{1-6}$ In addition, it is frequently noted in patients with known or subsequently new diagnosis of inflammatory bowel disease (IBD). ${ }^{7-9}$ However, there are few case reports and case series of immunocompetent patients without steroid use. ${ }^{10-16}$

The number of CMV colitis cases involving immunocompetent patients has noticeably increased in our hospital over the past few decades. However, the only meta-analysis study of such cases, involving 44 patients with immunomodulating comorbidities, and in whom the need for surgical intervention negatively influenced survival, was published in $2005 .{ }^{17}$ The differences between immunocompetent and immunocompromised patients and the factors affecting their in-hospital mortality were unknown.
Correspondence: Cheng-Tang Chiu Department of Gastroenterology and Hepatology, Linkou Chang Gung Memorial Hospital, 5 Fu-Hsin Street, Queishan, Taoyuan 333, Taiwan

Tel +886 3328 |200x8I0|

Fax +88633272236

Email ctchiu0508@gmail.com 


\section{Patients and methods}

\section{Patients}

Our investigation was approved by Chang Gung Medical Foundation Institutional Review Board on February 20, 2017 (approval document No 201700193B0 "Clinical presentations and outcome of cytomegalovirus infection in gastrointestinal tract") during February 01, 2017-February 01, 2018. Informed consent from individual patients to review the medical records covering patients' data was not required for retrospective studies with the electronic medical record system, by Chang Gung Medical Foundation Institutional Review Board. The study protocol conformed to the ethical guidelines of the 1975 Declaration of Helsinki, as reflected in a priori approval by the institution's human research committee.

We retrieved the clinical data of all patients who had pathologically proven CMV colitis from the pathology database in Linkou Chang Gung Memorial Hospital between April 2002 and December 2016. All patients in the study had pathologic specimens obtained by sigmoidoscopy, colonoscopy, or surgical resection. CMV colitis was diagnosed by both $\mathrm{CMV}$ inclusion bodies and positive immunohistochemistry (IHC) staining, which was performed with a monoclonal antibody directed against the CMV pp65 antigen (Novocastra ${ }^{\mathrm{TM}}$ lyophilized mouse monoclonal antibody; Leica Microsystems, Wetzlar, Germany), as illustrated in Figure 1.

Steroids are generally considered to be nonspecific immunosuppressants in most studies and textbooks, ${ }^{12,17,18}$ and the definition of immunocompromised patients in this study is the same as in most reviews or case reports of CMV colitis, ${ }^{9,17}$ except for one previous report. ${ }^{6}$ Patients were defined as immunocompromised if they had experienced solid organ or bone marrow transplantation, HIV infection, immunosuppressive drug use (including steroids [peroral or intravenous route, $\geq 20 \mathrm{mg}$ per day of prednisone or equivalent for $>2$ weeks]), or chemotherapeutic agent use within 6 months. All other patients were considered immunocompetent.

\section{Clinical data collection}

For this study, we collected clinical data on a considerable range of presenting symptoms, treatment duration, and outcome parameters, as well as on the following: age, gender, patient source, admission date, diagnostic date, recurrence date, death or last follow-up, general condition within 1 week before diagnosis, underlying diseases, medication history, total white blood cell (WBC) count, absolute neutrophil count (ANC), absolute lymphocyte count (ALC), hemoglobin (Hb), platelet (PLT) count, creatinine (Cr), aspartate aminotransferase (AST), alanine aminotransferase (ALT), bilirubin, albumin, C-reactive protein (CRP), CMV pp65 antigenemia, CMV DNA (226 bp segment on glycoprotein B gene, LightMix ${ }^{\circledR}$ Kit human Cytomegalovirus; TIB Molbiol, Berlin, Germany), CMV serology, and Clostridium difficile infection (BD MAX ${ }^{\mathrm{TM}}$ Cdiff Assay; GeneOhm Sciences Canada Inc, Quebec City, QC, Canada). Regarding the classification, "patient source," the designation of intensive care unit (ICU) presents patients who were admitted into the ICU before or during diagnosis in the same admission. "Requisite days of diagnosis" was defined as the duration between admission and diagnosis of CMV colitis. In addition, "recurrence" was defined as the finding of relapsed symptoms and signs with positive IHC stain after the patient had completed antiviral treatment and shown clinical improvement.
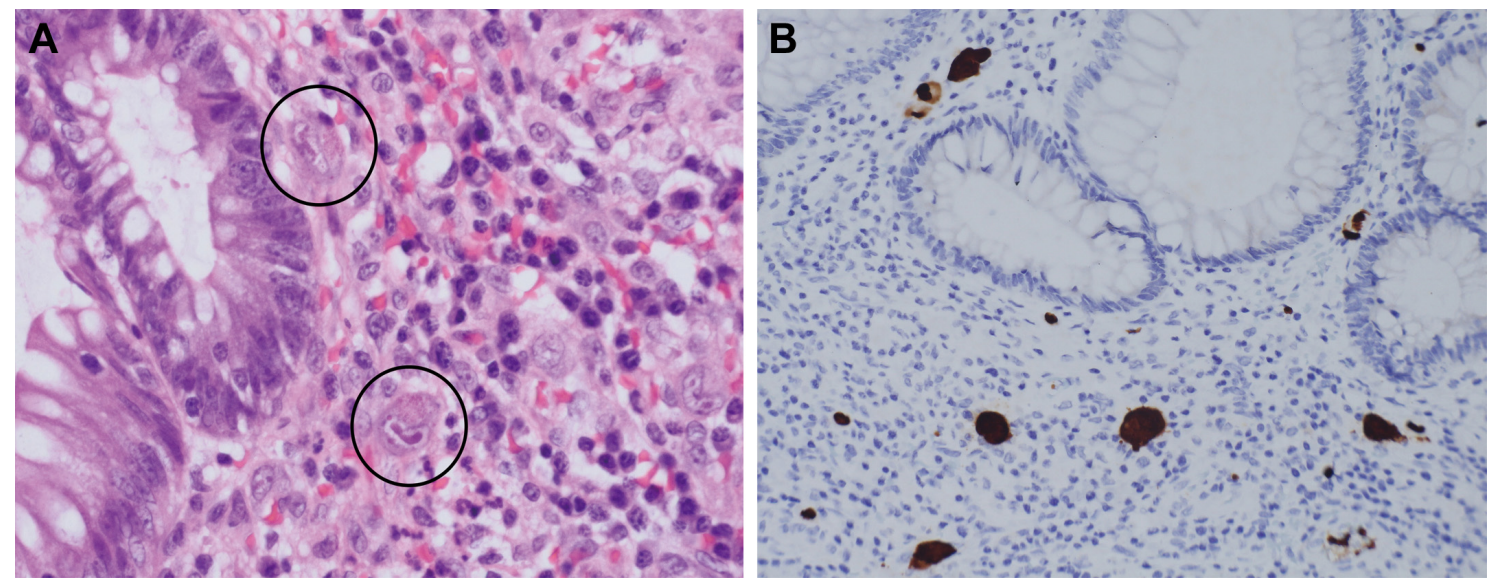

Figure I Pathological presentations of CMV colitis.

Notes: CMV colitis was diagnosed by both CMV inclusion bodies and positive IHC staining in the colonic tissue. (A) H\&E stain (40× objective) showed typical intranuclear (owl's eye) and intracytoplasmic (eosinophilic punctiform) CMV inclusions within the circles. (B) IHC stain (20× objective) was performed with I:200 diluted Novocastra ${ }^{\text {TM }}$ lyophilized mouse monoclonal antibody against CMV pp65 antigen and showed strong focal CMV immunoreactivity with brownish areas.

Abbreviations: CMV, cytomegalovirus; H\&E, hematoxylin and eosin; IHC, immunohistochemistry. 


\section{Statistical analysis}

To analyze the differences between the two groups of patients, independent $t$-tests and Mann-Whitney $U$ test were used to compare the continuous variables. $\chi^{2}$ and Fisher's exact tests were employed for the categorical variables. Numerical data were presented as mean \pm standard deviation, while categorical data were expressed as absolute numbers and percentages. Logistic regression model was used to identify the independent risk factors for in-hospital mortality. When any factor was found to be significant, via a $P$-value $<0.05$ in a univariate analysis, it was put into a multivariate model. The results are presented as odds ratio (OR), 95\% $\mathrm{CI}$, and $P$-values. If the continuous variables had clinical significance in the multivariate approach, we modified them into dichotomous variables using the optimal cutoff point as determined by receiver operating characteristic (ROC) curves using the Youden index for further overall survival (OS) evaluation with Kaplan-Meier survival curve analysis, employing a log-rank test. The results were considered to indicate a statistically significant difference when the respective $P$-values were $<0.05$. All statistical calculations were performed using SPSS software, version 21 (IBM Corporation, Armonk, NY, USA).

\section{Results}

\section{Baseline clinicopathological characteristics}

Of the total 69 patients enrolled in the study, 42 (60.87\%) were immunocompetent and the remainder were immunocompromised. Three of them had pathologically proven recurrence at the 38th, 63th, and 390th day after the first diagnosis (recurrence rate $=4.35 \%$ ); they received intravenous ganciclovir treatment ( 10 days, 10 days, and 37 days) and initially clinical symptoms improved. The 44-year-old patient with recurrence on the 38th day had HIV infection. The other two were immunocompetent patients $>70$ years old, with multiple comorbidities and critical illness in both CMV colitis episodes.

The average age of all patients was $59.42 \pm 20.11$ years. As far as general conditions were concerned, $>50 \%$ patients in this study had sepsis. Both shock and respiratory failure occurred in $31.9 \%$ patients, as summarized in Table 1 .

Among the eight patients with IBD after their episode of CMV colitis, two were subsequently diagnosed with ulcerative colitis and one with Crohn's disease. In the systemic lupus erythematosus patients, the $\mathrm{C} 3$ and $\mathrm{C} 4$ levels were $57.40 \pm 17.52$ and $15.9 \pm 4.45 \mathrm{mg} / \mathrm{dL}$, respectively. Two patients had undergone both renal and liver transplantation under mycophenolate, cyclosporine, and steroid use. Several solid organ malignancies were noted among patients in the study: rectal adenocarcinoma, ureteral urothelial carcinoma, hepatocellular carcinoma, and pancreatic head poorly differentiated adenocarcinoma. Two patients with hematological malignancies were diagnosed with peripheral $\mathrm{T}$ cell lymphoma and multiple myeloma. There were eight HIV patients in the study, and their average CD4 count was $50.86 \pm 89.80$ cells $/ \mathrm{mm}^{3}$. Five of them $(62.5 \%)$ were diagnosed with HIV due to CMV colitis events, and three HIV patients (37.5\%) were undergoing high levels of active antiretroviral therapy at diagnosis. No CMV colitis patients with bone marrow transplantation were recorded in the study. Other clinical characteristics and laboratory data are summarized in Table 1.

\section{Immunocompetent patients with CMV colitis have more comorbidities}

There were more patients with advanced age ( $\geq 65$ years), ICU admission, coronary artery disease, and hypertension in the immunocompetent group than those in the immunocompromised group. The former also had significantly longer hospital stays $(247.70 \pm 795.02$ days, $40.00 \pm 24.56$ days, $P=0.023)$. There were no significant differences between these two groups in the laboratory data, except for lower ALC levels in the immunocompromised group.

\section{Different presentations of CMV colitis in different immunological status}

The first presentation of CMV colitis in patients included melena $(n=29,42 \%)$, diarrhea $(n=28,40.6 \%)$, and abdominal pain $(n=8,11.6 \%)$. Four of these cases also first presented as positive stool occult blood (two cases), colonoscopy health examination (one case), and regular follow-up after colectomy due to colon cancer (one case). Although diarrhea (49.3\%) and melena (46.4\%) were the first and second most common symptoms in patients with CMV colitis, immunological status revealed that melena $(47.6 \%)$ was the most common first presentation in immunocompetent patients and diarrhea (59.3\%) in immunocompromised ones. The details are summarized in Table 2 .

\section{CMV reactivation plays a major role in CMV colitis}

The gold standard for diagnosing CMV colitis is histopathology, and negative plasma or whole blood polymerase chain reaction (PCR) does not exclude CMV colitis. In this study, most patients had incomplete laboratory data regarding 
Table I Baseline characteristics of patients with CMV colitis

\begin{tabular}{|c|c|c|c|c|}
\hline Characteristics & $\begin{array}{l}\text { Overall } \\
(n=69)\end{array}$ & $\begin{array}{l}\text { Immunocompetent } \\
(n=42)\end{array}$ & $\begin{array}{l}\text { Immunocompromised } \\
(n=27)\end{array}$ & $P$-value \\
\hline Age, years & $59.42 \pm 20.11$ & $64.40 \pm 19.36$ & $51.67 \pm 19.08$ & $0.009 *$ \\
\hline Age $\geq 65$ years & $33(47.8 \%)$ & $26(61.9 \%)$ & $7(25.9 \%)$ & $0.004 *$ \\
\hline Gender (male/female) & $42 / 27$ & $24 / 18$ & $18 / 9$ & 0.429 \\
\hline \multicolumn{5}{|l|}{ Patient source } \\
\hline Outpatient department & $6(8.7 \%)$ & 5 (II.9\%) & I (3.7\%) & 0.392 \\
\hline Intensive care unit & $27(39.1 \%)$ & $21(50 \%)$ & $6(22.2 \%)$ & $0.021^{*}$ \\
\hline Requisite days of diagnosis & $22.69 \pm 19.18$ & $23.5 I \pm 20.67$ & $21.42 \pm 16.98$ & 0.807 \\
\hline Hospital stay (days) & $|6| .98 \pm 6|4.7|$ & $247.70 \pm 795.02$ & $40.00 \pm 24.56$ & $0.023 *$ \\
\hline \multicolumn{5}{|l|}{ General condition } \\
\hline Sepsis & $46(66.7 \%)$ & $28(66.7 \%)$ & $18(66.7 \%)$ & 1.000 \\
\hline Shock & 22 (31.9\%) & $13(3 \mid \%)$ & $9(33.3 \%)$ & 0.836 \\
\hline Respiratory failure & $22(31.9 \%)$ & $15(35.7 \%)$ & $7(25.9 \%)$ & 0.394 \\
\hline \multicolumn{5}{|l|}{ Underlying diseases } \\
\hline Inflammatory bowel disease & $8(11.6 \%)$ & $7(16.7 \%)$ & I (3.7\%) & 0.136 \\
\hline Ulcerative colitis & 7 (10.1\%) & $6(14.3 \%)$ & I (3.7\%) & 0.233 \\
\hline Crohn's disease & I (I.4\%) & I (2.4\%) & $0(0 \%)$ & 1.000 \\
\hline Systemic lupus erythematosus & $5(7.2 \%)$ & I (2.4\%) & $4(14.8 \%)$ & 0.073 \\
\hline Solid organ transplantation & $2(2.9 \%)$ & $0(0 \%)$ & $2(7.4 \%)$ & 0.150 \\
\hline Solid organ malignancy & $4(5.8 \%)$ & $\mathrm{I}(2.4 \%)$ & $3(11.1 \%)$ & 0.292 \\
\hline Hematological malignancy & $2(2.9 \%)$ & $0(0 \%)$ & $2(7.4 \%)$ & 0.150 \\
\hline Liver cirrhosis & $4(5.8 \%)$ & $2(4.8 \%)$ & $2(7.4 \%)$ & $0.64 I$ \\
\hline \multicolumn{5}{|l|}{ Cardiovascular disease } \\
\hline Coronary artery disease & $8(11.6 \%)$ & $8(19.0 \%)$ & $0(0 \%)$ & $0.019 *$ \\
\hline Congestive heart failure & 7 (10.1\%) & $5(11.9 \%)$ & $2(7.4 \%)$ & 0.697 \\
\hline COPD & $3(4.3 \%)$ & $2(4.8 \%)$ & I (3.7\%) & 1.000 \\
\hline \multicolumn{5}{|l|}{ Renal disease } \\
\hline Acute kidney injury & $21(30.4 \%)$ & 14 (33.3\%) & 7 (25.9\%) & 0.514 \\
\hline Chronic kidney disease & $13(18.8 \%)$ & $6(14.3 \%)$ & $7(25.9 \%)$ & 0.228 \\
\hline End-stage renal disease & $8(11.6 \%)$ & $6(14.3 \%)$ & $2(7.4 \%)$ & 0.467 \\
\hline \multicolumn{5}{|l|}{ Neurological disease } \\
\hline Stroke & $10(14.5 \%)$ & $8(19.0 \%)$ & $2(7.4 \%)$ & 0.295 \\
\hline Parkinsonism & $6(8.7 \%)$ & 5 (11.9\%) & I (3.7\%) & 0.392 \\
\hline Epilepsy & 7 (10.14\%) & $6(14.29 \%)$ & I (3.7\%) & 0.233 \\
\hline Thyroid disease & $4(5.8 \%)$ & $3(7.1 \%)$ & I (3.7\%) & 1.000 \\
\hline Diabetes mellitus & $21(30.4 \%)$ & $15(35.7 \%)$ & $6(22.2 \%)$ & 0.235 \\
\hline Hypertension & $23(33.3 \%)$ & $19(45.2 \%)$ & $4(14.8 \%)$ & $0.010 *$ \\
\hline HIV infection & $8(11.6 \%)$ & $0(0 \%)$ & $8(29.6 \%)$ & \\
\hline \multicolumn{5}{|l|}{ Immunosuppressive medication } \\
\hline Chemotherapy & I (I.4\%) & $0(0 \%)$ & I (3.7\%) & \\
\hline Steroids & $18(26.1 \%)$ & $0(0 \%)$ & $18(66.7 \%)$ & \\
\hline Steroid use over I month before diagnosis of CMV colitis & $7(10.1 \%)$ & $0(0 \%)$ & $7(25.9 \%)$ & \\
\hline Total prednisolone dosage within I month before diagnosis & $993.06 \pm 1,338.72$ & 0 & $993.06 \pm 1,338.72$ & \\
\hline \multicolumn{5}{|l|}{ of CMV colitis, $m g(n=18)$} \\
\hline Other immunosuppressants & $6(8.7 \%)$ & $0(0 \%)$ & $6(22.2 \%)$ & \\
\hline \multicolumn{5}{|l|}{ Laboratory data } \\
\hline Total WBC count $(/ \mu \mathrm{L})$ & $8,|87.3| \pm 4,240.07$ & $8,437.80 \pm 3,828.82$ & $7,792.3 \mathrm{I} \pm 4,872.53$ & 0.344 \\
\hline ANC $(/ \mu \mathrm{L})$ & $6,309.84 \pm 3,974.94$ & $6,350.70 \pm 3,768.83$ & $6,246.97 \pm 4,349.18$ & 0.823 \\
\hline $\operatorname{ALC}(/ \mu \mathrm{L})$ & $\mathrm{I}, 134.22 \pm 843.45$ & $1,297.15 \pm 925.25$ & $883.57 \pm 637.46$ & $0.045^{*}$ \\
\hline Hemoglobin level (g/dL) & $10.66 \pm 2.24$ & $10.97 \pm 2.42$ & $10.18 \pm 1.85$ & 0.203 \\
\hline Platelet count $\left(\times 1,000 / \mathrm{mm}^{3}\right)$ & $239.38 \pm 113.48$ & $243.70 \pm 92.69$ & $232.73 \pm 141.43$ & 0.355 \\
\hline Creatinine (mg/dL) & $1.88 \pm 1.78$ & $1.94 \pm 1.66$ & $1.80 \pm 1.97$ & 0.374 \\
\hline ALT (IU/L) & $23.90 \pm 16.64$ & $20.57 \pm 12.39$ & $28.56 \pm 20.61$ & 0.179 \\
\hline Bilirubin (mg/dL) & $1.26 \pm 1.84$ & $1.22 \pm 1.66$ & $1.32 \pm 2.12$ & 0.598 \\
\hline Albumin $(g / d L)$ & $2.64 \pm 0.66$ & $2.63 \pm 0.67$ & $2.65 \pm 0.65$ & 0.967 \\
\hline C-reactive protein $(\mathrm{mg} / \mathrm{dL})$ & $71.14 \pm 72.91$ & $60.90 \pm 56.15$ & $87.72 \pm 93.19$ & 0.591 \\
\hline Bacteremia & $16(23.2 \%)$ & $8(19 \%)$ & $8(29.6 \%)$ & 0.393 \\
\hline Fungemia & $5(7.2 \%)$ & $3(7.1 \%)$ & $2(7.4 \%)$ & 1.000 \\
\hline
\end{tabular}

Notes: $* P<0.05$. Numerical data were presented as mean \pm standard deviation, while categorical data were expressed as absolute number (percentage). Requisite days of diagnosis was defined as the duration between admission and diagnosis of CMV colitis.

Abbreviations: ALC, absolute lymphocyte count; ALT, alanine aminotransferase; ANC, absolute neutrophil count; CMV, cytomegalovirus; COPD, chronic obstructive pulmonary disease; HIV, human immunodeficiency virus; WBC, white blood cell. 
Table 2 Clinical presentations of CMV colitis

\begin{tabular}{|c|c|c|c|c|}
\hline Characteristics & $\begin{array}{l}\text { Overall } \\
(n=69)\end{array}$ & $\begin{array}{l}\text { Immunocompetent } \\
(n=42)\end{array}$ & $\begin{array}{l}\text { Immunocompromised } \\
(n=27)\end{array}$ & $P$-value \\
\hline \multicolumn{5}{|l|}{ Chief complaint } \\
\hline Gl symptoms & 31 (44.9\%) & $19(45.2 \%)$ & $12(44.4 \%)$ & 0.948 \\
\hline Heart symptoms & $5(7.2 \%)$ & $4(9.5 \%)$ & I (3.7\%) & $0.64 I$ \\
\hline Sepsis & $24(34.8 \%)$ & $13(31 \%)$ & II (40.7\%) & 0.405 \\
\hline \multicolumn{5}{|l|}{ First presentation } \\
\hline Melena & 29 (42\%) & $20(47.6 \%)$ & $9(33.3 \%)$ & $0.24 I$ \\
\hline Diarrhea & $28(40.6 \%)$ & $12(28.6 \%)$ & $16(59.3 \%)$ & $0.01 I^{*}$ \\
\hline Pain & $8(11.6 \%)$ & $7(16.7 \%)$ & I (3.7\%) & 0.136 \\
\hline \multicolumn{5}{|l|}{ Symptoms } \\
\hline Melena & $32(46.4 \%)$ & $22(52.4 \%)$ & $10(37 \%)$ & 0.212 \\
\hline Diarrhea & $34(49.3 \%)$ & $15(35.7 \%)$ & 19 (70.4\%) & $0.005^{*}$ \\
\hline Pain & $16(23.2 \%)$ & $12(28.6 \%)$ & $4(14.8 \%)$ & 0.248 \\
\hline Fever & $33(47.8 \%)$ & 17 (40.5\%) & $16(59.3 \%)$ & 0.127 \\
\hline \multicolumn{5}{|l|}{ Viral markers } \\
\hline CMV pp65 antigenemia $(n=33,18,15)$ & $22(66.7 \%)$ & $10(55.6 \%)$ & $12(80 \%)$ & 0.266 \\
\hline Positive (/500,000 PMN) & $87.23 \pm 183.46$ & $58.00 \pm 144.48$ & $111.58 \pm 213.90$ & 0.346 \\
\hline CMV PCR positive $(n=8,4,4)$ & $4(50 \%)$ & $2(50 \%)$ & $2(50 \%)$ & 1.000 \\
\hline CMV IgG positive $(n=38,23,15)$ & $36(94.7 \%)$ & $22(95.7 \%)$ & 14 (93.3\%) & 1.000 \\
\hline CMV IgM positive $(n=38,23,15)$ & 7 (I8.4\%) & $5(21.7 \%)$ & $2(13.3 \%)$ & $0.68 I$ \\
\hline \multicolumn{5}{|l|}{ Medical treatment } \\
\hline Ganciclovir or valganciclovir & $50(72.46 \%)$ & $30(7 \mathrm{I} .43 \%)$ & 20 (74.07\%) & 0.810 \\
\hline Ganciclovir, iv, only & $28(40.6 \%)$ & I8 (42.9\%) & $10(37.0 \%)$ & 0.631 \\
\hline Valganciclovir, po, only & $12(17.4 \%)$ & $6(14.3 \%)$ & $6(22.2 \%)$ & 0.396 \\
\hline Ganciclovir and valganciclovir & $10(14.5 \%)$ & $6(14.3 \%)$ & $4(14.8 \%)$ & 1.000 \\
\hline Duration (days) & $22.80 \pm 21.90$ & $22.13 \pm 11.05$ & $23.80 \pm 32.39$ & 0.069 \\
\hline \multicolumn{5}{|l|}{ Outcome } \\
\hline Perforation & $3(4.3 \%)$ & $2(4.8 \%)$ & I (3.7\%) & 1.000 \\
\hline Surgical & $8(11.6 \%)$ & $6(14.3 \%)$ & $2(7.4 \%)$ & 0.467 \\
\hline In-hospital mortality & $18(26.1 \%)$ & II (26.2\%) & $7(25.9 \%)$ & 0.981 \\
\hline Overall survival & 45 (65.22\%) & $28(66.67 \%)$ & $17(62.97 \%)$ & 0.753 \\
\hline
\end{tabular}

Notes: $* P<0.05$. Numerical data were presented as mean \pm standard deviation, while categorical data were expressed as absolute number (percentage).

Abbreviations: CMV, cytomegalovirus; GI, gastrointestinal; iv, intravenous; PCR, polymerase chain reaction; PMN, polymorphonuclear leukocyte; po, per oral.

CMV infection markers (Table 2). Regarding available data, $66.7 \%$ patients had CMV of pp65 antigenemia. The CMV IgG-positive rate $(94.7 \%)$ was far more than the CMV IgMpositive rate (18.4\%). Although there was no resultant CMV IgG data, CMV reactivation might play a major role in $\mathrm{CMV}$ colitis. However, it did not affect the mortality outcomes in our study.

\section{C. difficile coinfection does not worsen the outcome}

CMV colitis has been reported as one of the more significant risk factors for $C$. difficile colonization. ${ }^{19}$ However, no study has demonstrated the relationship between coinfection and mortality rate in this context. Four of our patients had evidence of $C$. difficile coinfection and they were equally distributed across the immunocompetent and immunocompromised groups. Although the incidence was higher in the immunocompromised group, it was not statistically significant.
Furthermore, $C$. difficile coinfection was not a risk factor for in-hospital mortality $(P=0.925)$.

\section{Clinical complications and endoscopic follow-up}

In our study cohort, three patients $(4.3 \%)$ had perforation and eight (11.6\%) needed surgical treatment due to perforation, massive bleeding, or abscess. During follow-up, three patients $(4.3 \%)$ had stricture and none of them had IBD. The in-hospital mortality and OS rates were $26.1 \%$ and $65.22 \%$, respectively.

Only 21 patients had endoscopic follow-up after clinical improvement, and none had an active colonic lesion. Eight patients repeated endoscopic examination due to progressive or recurrent signs and symptoms. Among these, two, one, one, and four patients were IBD cases, HIV host, without treatment (improved after treatment), and with progressive septic shock, respectively; subsequently, two died. 


\section{Early diagnosis of CMV colitis improves survival}

In univariate analyses, we found that ICU admission, requisite days of diagnosis, shock, respiratory failure, ALC, $\mathrm{Hb}, \mathrm{Cr}$, and $\mathrm{CRP}$ were statistically significantly associated with in-hospital mortality. However, in multivariate logistic analyses, the requisite days of diagnosis was the only significant independent predictor of in-hospital mortality (OR $1.075,95 \%$ CI $1.005-1.149, P=0.035)$. We then determined the optimal cutoff point of 9 days from admission by further evaluation with ROC curves and the Youden index. Using the Kaplan-Meier survival curve analysis, we found that the patients with diagnosis before 9 days had a significantly better OS rate (log rank $P=0.018$ ), as shown in Figure 2 . In addition, the patients with immunocompromised status or steroid use had lower in-hospital mortality rates or worse OS outcomes, as illustrated in Figure 3, with the details summarized in Table 3.

\section{Discussion}

Although CMV colitis was considered rare in immunocompetent hosts, ${ }^{20-22}$ the recent increase in the number of published case reports of this type, especially involving patients with

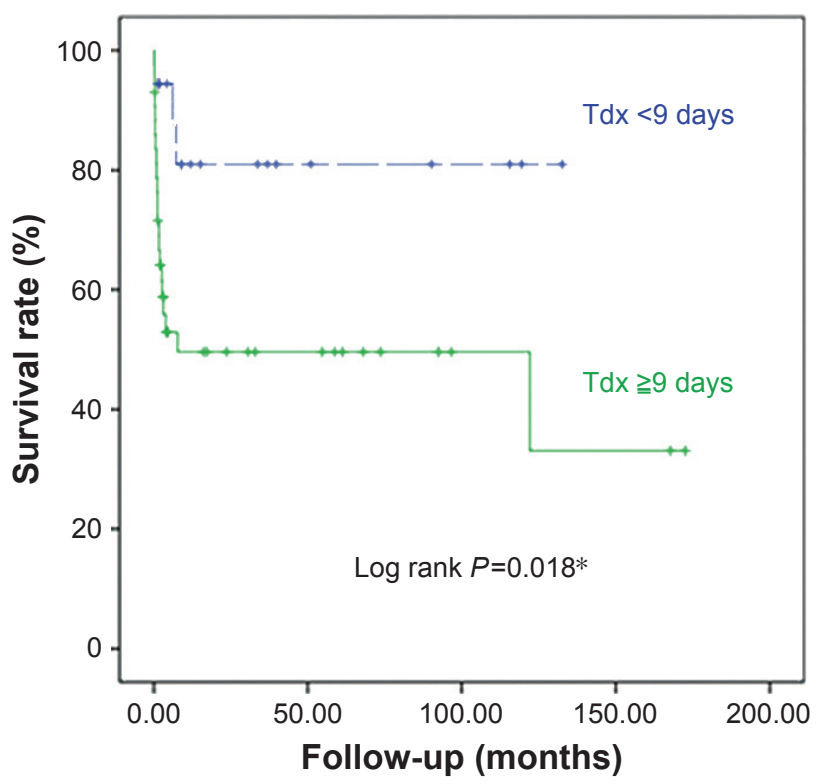

Figure 2 The survival curves of CMV patients with different requisite days of diagnosis.

Notes: $* P<0.05$. The number of requisite days of diagnosis (defined as the duration between admission and diagnosis of CMV colitis [Tdx]) from admission was the only predictor of in-hospital mortality. With the ROC curve and Youden index, we obtained the cutoff point of 9 days from a Kaplan-Meier survival curve analysis. The patients with diagnosis before 9 days from admission (dashed line) had significantly better OS than the ones who were diagnosed as CMV colitis after 9 days from admission (solid line).

Abbreviations: CMV, cytomegalovirus; OS, overall survival; ROC, receiver operating characteristic.
IBD, critical illness, and end-stage renal disease, drew our attention and concern. ${ }^{8,16,23-25}$ However, we could find no study that could predict mortality in such patients. Therefore, we designed the current study to improve this situation.

\section{CMV colitis in immunocompetent patients}

In this study, there were 42 immunocompetent patients (60.87\%) with CMV colitis. This observation notably revealed CMV colitis was not rare in immunocompetent patients. However, 19 of these patients (45.24\%) had comorbidities associated with immune dysfunction, including diabetes mellitus, liver cirrhosis, and end-stage renal disease. Ten others had critical conditions, such as ICU admission and respiratory failure or shock, and only 13 patients (30.95\%) had no clinical conditions with possible immune dysfunction. Besides, recent studies have shown that mucosal defects can increase the risk of CMV infection. ${ }^{9,26}$ If we further excluded patients with IBD, only eight cases were left. Therefore, most (80.95\%) of the immunocompetent patients with CMV colitis had comorbidities associated with immune dysfunction, critical illness, or IBD. In addition, $61.9 \%$ of these patients were $\geq 65$ years old, and they potentially had more comorbidities, higher risk for critical illness, and poorer immunological suppression for CMV primary or reactive infection.

The only earlier study of this subject (in 2005), involving 44 such patients, found that $75.2 \%$ of them indeed had immunomodulating conditions, including pregnancy, renal failure, diabetes mellitus, and untreated nonhematological malignancies. ${ }^{17}$ These findings were mostly compatible with ours, although most of their 44 patients were $<55$ years old, while $61.9 \%$ of ours were $\geq 65$ years old and had multiple comorbidities.

Although diagnostic-based patient selection and treatment was believed integral to optimal outcomes in patients with CMV colitis who had IBD, ${ }^{27}$ no evidence showed a similar benefit for other immunocompetent patients. Our review of severe CMV infection in immunocompetent patients revealed inconclusive results because the improvement observed in some of the patients receiving antiviral agents might be related to the typically self-limiting course of the disease rather than to any antiviral effect. ${ }^{28}$ The other concern was to balance between the potential morbidity of CMV colitis and the risk of medical toxicity, especially bone marrow suppression and renal insufficiency. Although $71.43 \%$ of immunocompetent patients were given antiviral treatments with an average duration of 22.13 days in this 

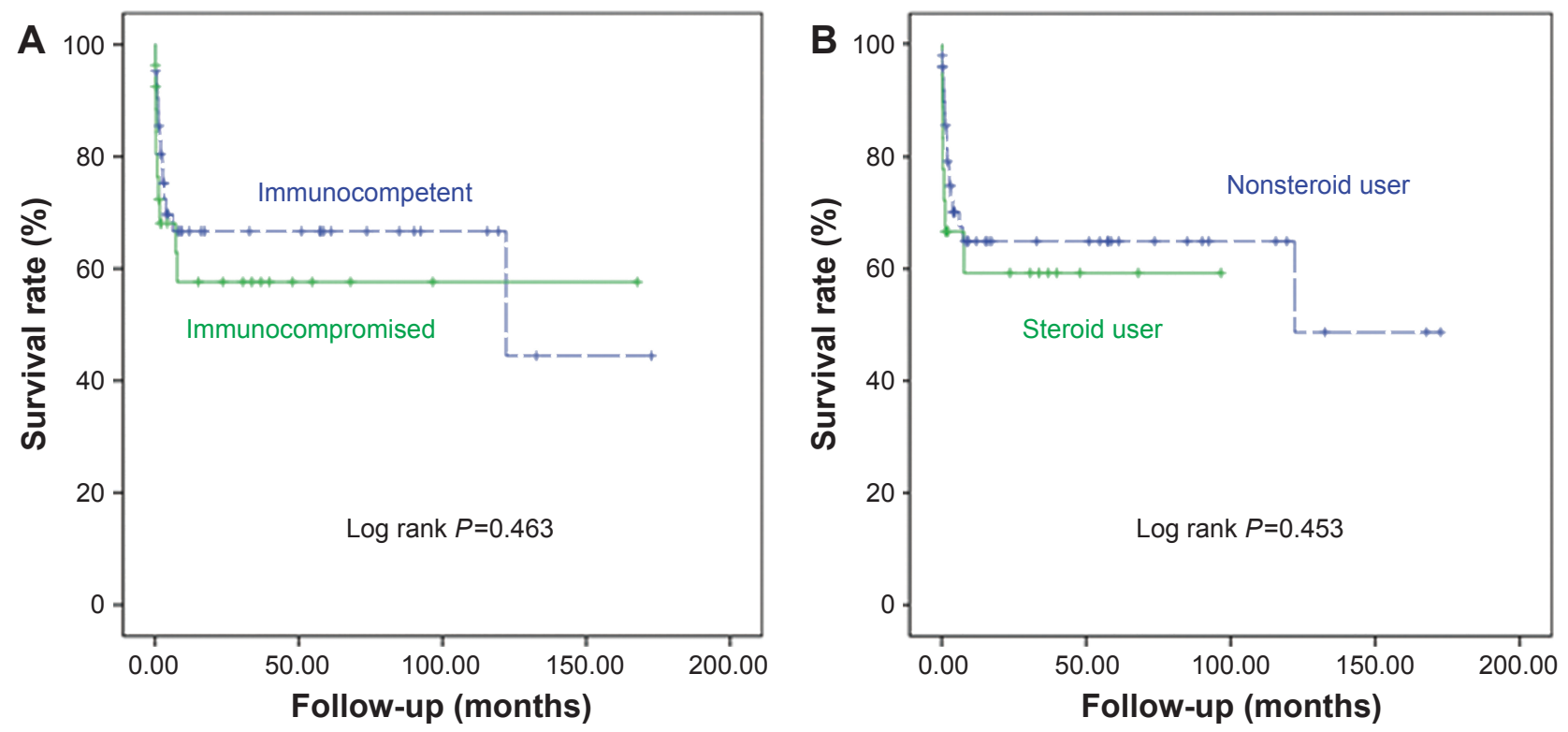

Figure 3 The survival curves of CMV colitis patients with different immunological status and steroid use.

Notes: (A) Immunocompetent (dashed line) and immunocompromised (solid line) patients with CMV colitis had similar OS according to Kaplan-Meier survival curve analysis. (B) Nonsteroid users (dashed line) and steroid users (solid line) with CMV colitis had similar OS according to a Kaplan-Meier survival curve analysis.

Abbreviations: CMV, cytomegalovirus; OS, overall survival.

study, in-hospital mortality appeared to be unrelated to whether or not patients received such treatment.

\section{CMV colitis in immunocompromised patients}

Among our 27 immunocompromised patients, 66.7\% were high-dose steroid users with an average steroid dosage equal to using a total of $993.06 \mathrm{mg}$ of prednisolone within 28 days. In addition, among these 27 patients, we had only eight HIV cases, two solid organ transplantations, and two hematological malignancies. These numbers were lower than we expected. Better supportive care and experimental antiviral treatment before pathological diagnosis might explain this finding. Although antiviral treatment was suggested for the immunocompromised patients with CMV colitis, only $74.07 \%$ of them received antiviral treatment in the study. These immunocompromised patients had relatively low levels of WBC, ANC, and ALC, as well as Hb and PLT count; therefore, the bone marrow suppression effect of antiviral agents might be an important concern in medical treatment.

\section{The differences between immunocompetent and immunocompromised patients with CMV colitis}

In the immunocompetent group, the patients were older $(P=0.009)$ and had more ICU admissions $(P=0.021)$, longer hospital stays $(P=0.023)$, more comorbidities (especially coronary artery disease $[P=0.019]$, and hypertension $[P=0.010])$, and also a higher ALC $(P=0.045)$, as presented in Table 1 . These findings suggest that these patients actually had more comorbidities and worse general condition than our immunocompromised patients. However, the in-hospital mortality OS rates were similar, as detailed in Table 2 and Figure 3. This outcome might be due to more complications of immunosuppressants and immunocompromised diseases, such as HIV and hematological malignancy in the immunocompromised group, negatively influencing their survival rate.

It turned out that the most common first presentation was quite different in the two groups; melena in immunocompetent patients and diarrhea in the immunocompromised patients. The former group included more patients with ICU admission, multiple comorbidities, and medications, leading to a higher risk of bleeding, which might explain the finding. In contrast, for the immunocompromised group, a higher risk of opportunistic coinfection, such as $C$. difficile $(7.41 \%$ vs $4.76 \%$ ), might explain the higher percentage of diarrhea. If clinicians are more aware of the different presentations and their implications, early diagnosis of CMV colitis could lead to a lower in-hospital mortality rate.

The duration of antiviral therapy depended on the patient and the severity of the disease. In our study, immunocompromised patients received longer treatment durations 
Table 3 Analysis of the clinical factors associated with in-hospital mortality in all patients

\begin{tabular}{|c|c|c|c|}
\hline Characteristics & OR & $95 \% \mathrm{Cl}$ & $P$-value \\
\hline \multicolumn{4}{|l|}{ Univariate analysis } \\
\hline Age $\geq 65$ years & 2.071 & $0.691-6.209$ & 0.194 \\
\hline Gender (male/female) & 0.545 & $0.184-1.619$ & 0.275 \\
\hline Immunocompromised status & 0.986 & $0.328-2.969$ & 0.981 \\
\hline Intensive care unit admission & $6.87 \mid$ & $2.068-22.833$ & $0.002 *$ \\
\hline Requisite days of diagnosis & 1.034 & $1.002-1.066$ & $0.034 *$ \\
\hline \multicolumn{4}{|l|}{ General condition } \\
\hline Sepsis & $1.039 \times 10^{9}$ & $0.000->10^{12}$ & 0.998 \\
\hline Shock & 5.714 & $1.793-18.210$ & $0.003 *$ \\
\hline Respiratory failure & 4.062 & $1.309-12.610$ & $0.015^{*}$ \\
\hline Operation before diagnosis & 3.200 & $0.583-17.553$ & 0.180 \\
\hline \multicolumn{4}{|l|}{ Underlying diseases } \\
\hline Inflammatory bowel disease & 0.000 & 0.000 & 0.999 \\
\hline Systemic lupus erythematosus & 4.900 & $0.747-32.123$ & 0.098 \\
\hline Solid organ transplantation & 2.941 & $0.174-49.636$ & 0.454 \\
\hline Solid organ malignancy & $0.94 I$ & $0.092-9.671$ & 0.959 \\
\hline Hematological malignancy & $2.94 I$ & $0.174-49.636$ & 0.454 \\
\hline Liver cirrhosis & 0.941 & $0.092-9.67 \mid$ & 0.959 \\
\hline Chronic kidney disease & 2.067 & $0.576-7.421$ & 0.265 \\
\hline End-stage renal disease & 3.357 & $0.742-15.18 \mid$ & 0.116 \\
\hline Diabetes mellitus & 1.682 & $0.543-5.205$ & 0.367 \\
\hline HIV infection & 0.000 & $0.000->10^{12}$ & 0.999 \\
\hline \multicolumn{4}{|l|}{ Immunosuppressive medication } \\
\hline Immunosuppressant & 3.200 & $0.583-17.553$ & 0.180 \\
\hline Chemotherapy & $4.846 \times 10^{9}$ & $0.000->10^{12}$ & 1.000 \\
\hline Steroids & 1.124 & $0.336-3.764$ & 0.849 \\
\hline $\begin{array}{l}\text { Steroid use over I month } \\
\text { before diagnosis of CMV colitis }\end{array}$ & 2.350 & $0.472-11.708$ & 0.297 \\
\hline $\begin{array}{l}\text { Total prednisolone dosage } \\
\text { within I month before diagnosis } \\
\text { of } \mathrm{CMV} \text { colitis, } \mathrm{mg}(\mathrm{n}=18)\end{array}$ & 1.000 & $0.999-1.001$ & 0.775 \\
\hline \multicolumn{4}{|l|}{ Laboratory data } \\
\hline Total WBC count $(/ \mu \mathrm{L})$ & 1.000 & $1.000-1.000$ & 0.419 \\
\hline ANC $(/ \mu \mathrm{L})$ & 1.000 & $1.000-1.000$ & 0.254 \\
\hline $\mathrm{ALC}(/ \mu \mathrm{L})$ & 0.999 & $0.998-1.000$ & $0.018 *$ \\
\hline Hemoglobin level (g/dL) & 0.668 & $0.485-0.918$ & $0.013^{*}$ \\
\hline Platelet count $\left(\times 1,000 / \mathrm{mm}^{3}\right)$ & 0.995 & $0.990-1.001$ & 0.100 \\
\hline Creatinine (mg/dL) & 1.448 & $1.059-1.978$ & $0.020 *$ \\
\hline ALT (IU/L) & 0.995 & $0.958-1.033$ & 0.787 \\
\hline Bilirubin (mg/dL) & 1.370 & $0.965-1.944$ & 0.078 \\
\hline Albumin (g/dL) & 0.625 & $0.23 \mathrm{I}-1.687$ & 0.354 \\
\hline C-reactive protein (mg/dL) & 1.009 & $1.000-1.018$ & $0.047 *$ \\
\hline \multicolumn{4}{|l|}{ Viral markers } \\
\hline CMV pp65 antigenemia & 0.656 & $0.140-3.079$ & 0.593 \\
\hline CMV IgG positive & 0.286 & $0.016-5.095$ & 0.394 \\
\hline CMV IgM positive & 3.125 & $0.547-|7.84|$ & 0.200 \\
\hline C. difficile infection & 0.889 & $0.077-10.300$ & 0.925 \\
\hline $\begin{array}{l}\text { Ganciclovir or valganciclovir } \\
\text { treatment }\end{array}$ & 2.286 & $0.579-9.026$ & 0.238 \\
\hline Treatment duration & 0.989 & $0.953-1.026$ & 0.563 \\
\hline Surgical treatment & 1.840 & $0.392-8.630$ & 0.439 \\
\hline Perforation & $1.44 \mid$ & $0.123-16.920$ & 0.771 \\
\hline \multicolumn{4}{|l|}{ Multivariate analysis } \\
\hline Intensive care unit admission & 4.726 & $0.374-59.747$ & 0.230 \\
\hline Requisite days of diagnosis & 1.075 & $1.005-1.149$ & $0.035^{*}$ \\
\hline Shock & 4.905 & $0.596-40.362$ & 0.139 \\
\hline
\end{tabular}

(Continued)
Table 3 (Continued)

\begin{tabular}{llll}
\hline Characteristics & OR & $\mathbf{9 5 \%} \mathbf{C l}$ & P-value \\
\hline Respiratory failure & 0.661 & $0.053-8.269$ & 0.748 \\
ALC $(/ \mu \mathrm{L})$ & 0.998 & $0.996-1.00 \mathrm{I}$ & 0.157 \\
Hemoglobin level $(\mathrm{g} / \mathrm{dL})$ & 0.787 & $0.459-1.349$ & 0.383 \\
Creatinine $(\mathrm{mg} / \mathrm{dL})$ & 0.618 & $0.306-1.246$ & 0.179 \\
C-reactive protein $(\mathrm{mg} / \mathrm{dL})$ & 1.009 & $0.998-1.021$ & 0.106 \\
\hline
\end{tabular}

Notes: $* P<0.05$. Requisite days of diagnosis was defined as the duration between admission and diagnosis of CMV colitis.

Abbreviations: ALC, absolute lymphocyte count; ALT, alanine aminotransferase; ANC, absolute neutrophil count; $C$. difficile, Clostridium difficile; CMV, cytomegalovirus; HIV, human immunodeficiency virus; OR, odds ratio; WBC, white blood cell.

( $23.80 \pm 32.39$ vs $22.13 \pm 11.05$ days), but there was no statistically significant difference in in-hospital mortality $(P=0.069)$. The complication rate, in-hospital mortality, and OS were also not significantly different.

\section{The predictors of in-hospital mortality in CMV colitis patients}

Although early diagnosis reduced in-hospital mortality and improved OS, antiviral treatment did not have these effects. This result might be related to underlying disease and general conditions as well as the timing and toxicity of the treatment. Furthermore, the virus might be nonpathogenic; thus, antiviral treatment is unhelpful. ${ }^{27}$ Therefore, we should evaluate clinical conditions individually to decide on the use of antiviral agents instead of treating everyone alike.

\section{The limitations of the study}

Although this investigation was the largest single study of CMV colitis based on a histopathological diagnosis until now, a prospective study with more cases is still needed. Besides, better immunological biomarkers of immunocompetence, including complement and immunoglobulin level, will be our further study target.

\section{Conclusion}

Contrary to data published up to this point, we found that CMV colitis was not rare and that it could be fatal in immunocompetent hosts, especially those patients with advanced age, specific comorbidities associated with immune dysfunction, critical illness, or IBD. Melena was the most common symptom found in this class of patients, but in contrast, diarrhea was the most common symptom in those who were immunocompromised. Being alert to the different presentations can greatly help make an accurate early diagnosis and materially improve survival for CMV colitis patients. 


\section{Acknowledgments}

We would like to thank Prof Liang Kung-Hao, Liver Research Center, Linkou Chang Gung Memorial Hospital, Taoyuan, Taiwan, for providing statistical consultation. This research received no specific grant from any funding agency in the public, commercial, or not-for-profit sectors.

\section{Disclosure}

The authors report no conflicts of interest in this work.

\section{References}

1. Nakase H, Herfarth H. Cytomegalovirus colitis, cytomegalovirus hepatitis and systemic cytomegalovirus infection: common features and differences. Inflamm Intest Dis. 2016;1(1):15-23.

2. Lemonovich TL, Watkins RR. Update on cytomegalovirus infections of the gastrointestinal system in solid organ transplant recipients. Curr Infect Dis Rep. 2012;14(1):33-40.

3. Whitley RJ, Jacobson MA, Friedberg DN, et al. Guidelines for the treatment of cytomegalovirus diseases in patients with AIDS in the era of potent antiretroviral therapy: recommendations of an international panel. International AIDS Society-USA. Arch Intern Med. 1998;158(9): 957-969.

4. Matthes T, Kaiser L, Weber D, Kurt AM, Dietrich PY. Cytomegalovirus colitis - a severe complication after standard chemotherapy. Acta Oncol. 2002;41(7-8):704-706.

5. Hani MA, Ben Achour J, Zoghlami A, Najah N. La colite aiguë cytomégalovirus après greffe de moelle osseuse [Acute cytomegalovirus colitis after bone marrow transplantation]. Tunis Med. 2003;81(2): 145-148. French.

6. Ko JH, Peck KR, Lee WJ, et al. Clinical presentation and risk factors for cytomegalovirus colitis in immunocompetent adult patients. Clin Infect Dis. 2015;60(6):e20-e26.

7. Kim YS, Kim YH, Kim JS, et al. The prevalence and efficacy of ganciclovir on steroid-refractory ulcerative colitis with cytomegalovirus infection: a prospective multicenter study. J Clin Gastroenterol. 2012 46(1):51-56.

8. Weng MT, Tung CC, Lee YS, et al. Cytomegalovirus colitis in hospitalized inflammatory bowel disease patients in Taiwan: a referral center study. BMC Gastroenterol. 2017;17(1):28.

9. Khan TV, Toms C. Cytomegalovirus colitis and subsequent new diagnosis of inflammatory bowel disease in an immunocompetent host: a case study and literature review. Am J Case Rep. 2016;17:538-543.

10. Einbinder Y, Wolf DG, Pappo O, Migdal A, Tsvang E, Ackerman Z. The clinical spectrum of cytomegalovirus colitis in adults. Aliment Pharmacol Ther. 2008;27(7):578-587.

11. Paparoupa M, Schmidt V, Weckauf H, Ho H, Schuppert F. CMV colitis in immunocompetent patients: 2 cases of a diagnostic challenge. Case Rep Gastrointest Med. 2016;2016:4035637.
12. Inayat $\mathrm{F}$, Hussain Q, Shafique K, Tasleem SH, Hurairah A. Cytomegalovirus colitis in immunocompetent patients. Cureus. 2016;8(11):e869.

13. Harano Y, Kotajima L, Arioka H. Case of cytomegalovirus colitis in an immunocompetent patient: a rare cause of abdominal pain and diarrhea in the elderly. Int J Gen Med. 2015;8:97-100.

14. Goodman AL, Murray CD, Watkins J, Griffiths PD, Webster DP. CMV in the gut: a critical review of CMV detection in the immunocompetent host with colitis. Eur J Clin Microbiol Infect Dis. 2015;34(1):13-18.

15. Klauber E, Briski LE, Khatib R. Cytomegalovirus colitis in the immunocompetent host: an overview. Scand J Infect Dis. 1998;30(6): $559-564$.

16. Siciliano RF, Castelli JB, Randi BA, Vieira RD, Strabelli TM. Cytomegalovirus colitis in immunocompetent critically ill patients. Int $J$ Infect Dis. 2014;20:71-73.

17. Galiatsatos P, Shrier I, Lamoureux E, Szilagyi A. Meta-analysis of outcome of cytomegalovirus colitis in immunocompetent hosts. Dig Dis Sci. 2005;50(4):609-616.

18. Azzi J, Milford EL, Sayegh MH, Chandraker A. Transplantation in the treatment of renal failure. In: Kasper D, Fauci A, Hauser S, Longo D, Jameson JL, Loscalzo J, editors. Harrison's Principles of Internal Medicine. 19 ed. New York, NY: McGraw-Hill Education; 2015.

19. Hutin Y, Casin I, Lesprit P, et al. Prevalence of and risk factors for Clostridium difficile colonization at admission to an infectious diseases ward. Clin Infect Dis. 1997;24(5):920-924.

20. Siegal DS, Hamid N, Cunha BA. Cytomegalovirus colitis mimicking ischemic colitis in an immunocompetent host. Heart Lung. 2005;34(4): 291-294.

21. Patra S, Samal SC, Chacko A, Mathan VI, Mathan MM. Cytomegalovirus infection of the human gastrointestinal tract. J Gastroenterol Hepatol. 1999;14(10):973-976.

22. Goodgame RW. Gastrointestinal cytomegalovirus disease. Ann Intern Med. 1993;119(9):924-935.

23. Nakashima K, Aoshima M, Suzuki F, Watanabe J, Otsuka Y. Cytomegalovirus colitis in a critically ill patient following severe legionella pneumonia with multiple organ failure. Intern Med. 2016;55(5):527-531.

24. Helgason KO, Raby SJ, Kamel HM, Laurenson IE, Templeton K, Walsh TS. Cytomegalovirus colitis in a critically ill patient following elective repair of an abdominal aortic aneurysm. Anaesth Intensive Care. 2008;36(1):107-109.

25. Kim SH, Kim YS, Kim HW, et al. A case of cytomegalovirus colitis in an immunocompetent hemodialysis patient. Hemodial Int. 2011; 15(2):297-300.

26. Hirayama Y, Ando T, Hirooka Y, et al. Characteristic endoscopic findings and risk factors for cytomegalovirus-associated colitis in patients with active ulcerative colitis. World J Gastrointest Endosc. 2016;8(6): 301-309.

27. Beswick L, Ye B, van Langenberg DR. Toward an algorithm for the diagnosis and management of CMV in patients with colitis. Inflamm Bowel Dis. 2016;22(12):2966-2976.

28. Rafailidis PI, Mourtzoukou EG, Varbobitis IC, Falagas ME. Severe cytomegalovirus infection in apparently immunocompetent patients: a systematic review. Virol J. 2008;5:47.
Therapeutics and Clinical Risk Management

\section{Publish your work in this journal}

Therapeutics and Clinical Risk Management is an international, peerreviewed journal of clinical therapeutics and risk management, focusing on concise rapid reporting of clinical studies in all therapeutic areas, outcomes, safety, and programs for the effective, safe, and sustained use of medicines. This journal is indexed on PubMed Central, CAS,

\section{Dovepress}

EMBase, Scopus and the Elsevier Bibliographic databases. The manuscript management system is completely online and includes a very quick and fair peer-review system, which is all easy to use. Visit $\mathrm{http}: / / \mathrm{ww}$.dovepress.com/testimonials.php to read real quotes from published authors. 